\title{
Search for Neutrinoless Double-Beta Decay with the Upgraded EXO-200 Detector
}

J. B. Albert, ${ }^{1}$ G. Anton, ${ }^{2}$ I. Badhrees, ${ }^{3, \dagger}$ P. S. Barbeau, ${ }^{4}$ R. Bayerlein, ${ }^{2}$ D. Beck, ${ }^{5}$ V. Belov, ${ }^{6}$ M. Breidenbach, ${ }^{7}$ T. Brunner, ${ }^{8,9}$ G. F. Cao, ${ }^{10}$ W. R. Cen,${ }^{10}$ C. Chambers, ${ }^{11}$ B. Cleveland, ${ }^{12,13}$ M. Coon,${ }^{5}$ A. Craycraft, ${ }^{11}$ W. Cree,${ }^{3}$ T. Daniels, ${ }^{7}$ M. Danilov, ${ }^{6, *}$ S. J. Daugherty, ${ }^{1}$ J. Daughhetee ${ }^{14}{ }^{14}$. Davis, ${ }^{7}$ S. Delaquis, ${ }^{7}$ A. Der Mesrobian-Kabakian, ${ }^{12}$ R. DeVoe, ${ }^{15}$ T. Didberidze, ${ }^{16,8}$ J. Dilling, ${ }^{9}$ A. Dolgolenko, ${ }^{6}$ M. J. Dolinski, ${ }^{17}$ W. Fairbank, Jr., ${ }^{11}$ J. Farine, ${ }^{12}$ S. Feyzbakhsh, ${ }^{18}$ P. Fierlinger, ${ }^{19}$

D. Fudenberg, ${ }^{15}$ R. Gornea, ${ }^{3,9}$ K. Graham, ${ }^{3}$ G. Gratta,${ }^{15}$ C. Hall, ${ }^{20}$ E. V. Hansen, ${ }^{17}$ J. Hoessl, ${ }^{2}$ P. Hufschmidt, ${ }^{2}$ M. Hughes,${ }^{16}$ A. Jamil, ${ }^{2,15}$ M. J. Jewell, ${ }^{15}$ A. Johnson, ${ }^{7}$ S. Johnston, ${ }^{18, \|}$ A. Karelin, ${ }^{6}$ L. J. Kaufman, ${ }^{1,9}$ T. Koffas, ${ }^{3}$ S. Kravitz, ${ }^{15}$ R. Krücken, ${ }^{9}$ A. Kuchenkov, ${ }^{6}$ K. S. Kumar, ${ }^{21}$ Y. Lan, ${ }^{9}$ D. S. Leonard, ${ }^{22}$ G. S. Li,${ }^{15}$ S. Li, ${ }^{5}$ C. Licciardi, ${ }^{3, *}$ Y. H. Lin, ${ }^{17}$ R. MacLellan, ${ }^{14}$ T. Michel, ${ }^{2}$ B. Mong, ${ }^{7}$ D. Moore ${ }^{23}$ K. Murray, ${ }^{8}$ R. Nelson,${ }^{24}$ O. Njoya, ${ }^{21}$ A. Odian, ${ }^{7}$ I. Ostrovskiy, ${ }^{16}$ A. Piepke, ${ }^{16}$ A. Pocar,${ }^{18}$ F. Retière, ${ }^{9}$ P. C. Rowson, ${ }^{7}$ J. J. Russell, ${ }^{7}$ S. Schmidt, ${ }^{2}$ A. Schubert, ${ }^{15}$ D. Sinclair, ${ }^{3,9}$ V. Stekhanov, ${ }^{6}$ M. Tarka, ${ }^{21}$ T. Tolba, ${ }^{10}$ R. Tsang, ${ }^{16, * *}$ P. Vogel, ${ }^{25}$ J.-L. Vuilleumier, ${ }^{26}$ M. Wagenpfeil, ${ }^{2}$ A. Waite, ${ }^{7}$ T. Walton, ${ }^{11}$ M. Weber, ${ }^{15}$ L. J. Wen, ${ }^{10}$ U. Wichoski, ${ }^{12}$ G. Wrede, ${ }^{2}$ L. Yang, ${ }^{5}$ Y.-R. Yen, ${ }^{17}$ O. Ya. Zeldovich, ${ }^{6}$ J. Zettlemoyer, ${ }^{1}$ and T. Ziegler ${ }^{2}$

\section{(EXO-200 Collaboration)}

\author{
${ }^{1}$ Physics Department and CEEM, Indiana University, Bloomington, Indiana 47405, USA \\ ${ }^{2}$ Erlangen Centre for Astroparticle Physics (ECAP), Friedrich-Alexander University Erlangen-Nürnberg, Erlangen 91058 , Germany \\ ${ }^{3}$ Physics Department, Carleton University, Ottawa, Ontario K1S 5B6, Canada \\ ${ }^{4}$ Department of Physics, Duke University, and Triangle Universities Nuclear Laboratory (TUNL), \\ Durham, North Carolina 27708, USA \\ ${ }^{5}$ Physics Department, University of Illinois, Urbana-Champaign, Illinois 61801, USA \\ ${ }^{6}$ Institute for Theoretical and Experimental Physics, Moscow, 117218, Russia \\ ${ }^{7}$ SLAC National Accelerator Laboratory, Menlo Park, California 94025, USA \\ ${ }^{8}$ Physics Department, McGill University, Montreal, Quebec, Canada \\ ${ }^{9}$ TRIUMF, Vancouver, British Columbia V6T 2A3, Canada \\ ${ }^{10}$ Institute of High Energy Physics, Beijing 100049, China \\ ${ }^{11}$ Physics Department, Colorado State University, Fort Collins, Colorado 80523, USA \\ ${ }^{12}$ Department of Physics, Laurentian University, Sudbury, Ontario P3E 2C6, Canada \\ ${ }^{13}$ SNOLAB, Sudbury, Ontario P3Y 1N2, Canada \\ ${ }^{14}$ Department of Physics, University of South Dakota, Vermillion, South Dakota 57069, USA \\ ${ }^{15}$ Physics Department, Stanford University, Stanford, California 94305, USA \\ ${ }^{16}$ Department of Physics and Astronomy, University of Alabama, Tuscaloosa, Alabama 35487, USA \\ ${ }^{17}$ Department of Physics, Drexel University, Philadelphia, Pennsylvania 19104, USA \\ ${ }^{18}$ Amherst Center for Fundamental Interactions and Physics Department, University of Massachusetts, \\ Amherst, Massachusetts 01003, USA \\ ${ }^{19}$ Technische Universität München, Physikdepartment and Excellence Cluster Universe, Garching 80805, Germany \\ ${ }^{20}$ Physics Department, University of Maryland, College Park, Maryland 20742, USA \\ ${ }^{21}$ Department of Physics and Astronomy, Stony Brook University, SUNY, Stony Brook, New York 11794, USA \\ ${ }^{22}$ IBS Center for Underground Physics, Daejeon 34047, Korea \\ ${ }^{23}$ Department of Physics, Yale University, New Haven, Connecticut 06511, USA \\ ${ }^{24}$ Waste Isolation Pilot Plant, Carlsbad, New Mexico 88220, USA \\ ${ }^{25}$ Kellogg Lab, Caltech, Pasadena, California 91125, USA \\ ${ }^{26}$ LHEP, Albert Einstein Center, University of Bern, Bern 3012, Switzerland
}

(Received 1 August 2017; revised manuscript received 6 October 2017; published 15 February 2018)

Results from a search for neutrinoless double-beta decay $(0 \nu \beta \beta)$ of ${ }^{136} \mathrm{Xe}$ are presented using the first year of data taken with the upgraded EXO-200 detector. Relative to previous searches by EXO-200, the energy resolution of the detector has been improved to $\sigma / E=1.23 \%$, the electric field in the drift region has been raised by $50 \%$, and a system to suppress radon in the volume between the cryostat and lead

\footnotetext{
Published by the American Physical Society under the terms of the Creative Commons Attribution 4.0 International license. Further distribution of this work must maintain attribution to the author(s) and the published article's title, journal citation, and DOI. Funded by $\operatorname{SCOAP}$.
} 
shielding has been implemented. In addition, analysis techniques that improve topological discrimination between $0 \nu \beta \beta$ and background events have been developed. Incorporating these hardware and analysis improvements, the median $90 \%$ confidence level $0 \nu \beta \beta$ half-life sensitivity after combining with the full data set acquired before the upgrade has increased twofold to $3.7 \times 10^{25} \mathrm{yr}$. No statistically significant evidence for $0 \nu \beta \beta$ is observed, leading to a lower limit on the $0 \nu \beta \beta$ half-life of $1.8 \times 10^{25} \mathrm{yr}$ at the $90 \%$ confidence level.

DOI: 10.1103/PhysRevLett.120.072701

Neutrinoless double-beta decay $(0 \nu \beta \beta)$, in which a nucleus with mass number $A$ and charge $Z$ undergoes the decay $(A, Z) \rightarrow(A, Z+2)+2 e^{-}$with the emission of no neutrinos [1], provides the most sensitive test of the Majorana nature of neutrinos [2]. While the corresponding two-neutrino double-beta decay $(2 \nu \beta \beta)$ has been observed for several nuclides [3], the observation of $0 \nu \beta \beta$ would provide direct evidence for a beyond-the-standard-model process that violates lepton number conservation and constrain the absolute neutrino mass scale [4]. Motivated by these implications, a variety of experiments are searching for $0 \nu \beta \beta$ in a number of nuclides, reaching half-life sensitivities in excess of $10^{25} \mathrm{yr}$ (see, e.g., Refs. [5-7]), with the most stringent for ${ }^{136} \mathrm{Xe}$ at $5.6 \times 10^{25} \mathrm{yr}[6]$.

EXO-200 is searching for $0 \nu \beta \beta$ in ${ }^{136} \mathrm{Xe}$ (see Refs. [5,8,9] for a detailed description). The detector consists of a cylindrical time projection chamber (TPC) filled with liquid xenon (LXe) enriched to $80.6 \%{ }^{136} \mathrm{Xe}$. The TPC is split into two drift regions by a common cathode, each with radius $\sim 18 \mathrm{~cm}$ and drift length $\sim 20 \mathrm{~cm}$. Energy depositions in the LXe produce both scintillation light and ionization. The ionization charge is read out after being drifted to crossed-wire planes at each anode by an electric field, while the scintillation light is collected by arrays of avalanche photodiodes (APDs) [10] located behind the wire planes. For each interaction, the location of the deposited charge in the directions perpendicular to the drift field ( $x$ and $y$ ) is determined from the wire signals. The $z$ position is reconstructed from the time delay between the prompt light signal and the delayed charge signals, using the measured ionization drift velocity [11]. The total energy deposited is determined from the combination of the charge and light signals, optimally accounting for their anticorrelation [12].

The LXe is housed in a thin-walled copper vessel, and surrounded by several layers of passive and active shielding, including $\sim 50 \mathrm{~cm}$ of HFE-7000 cryofluid [13] and $\sim 25 \mathrm{~cm}$ of lead in all directions [9]. A plastic scintillator muon veto surrounds the experiment on four sides $[8,14]$. The detector is located at the Waste Isolation Pilot Plant (WIPP) near Carlsbad, New Mexico, which provides an overburden of $1624_{-21}^{+22}$ meter water equivalent [14].

To reconstruct events in the TPC, charge and light signals are first grouped into individual energy deposits within each event. Events with a single reconstructed deposit are identified as "single-site" (SS), while events with multiple, spatially separated deposits are denoted as "multisite" (MS). This topological SS or MS classification has been used in previous EXO-200 analyses and provides discrimination between $\gamma$ backgrounds, which are primarily $\mathrm{MS}$, and the $0 \nu \beta \beta$ signal, which is primarily SS. A detailed detector Monte Carlo (MC) simulation based on GEANT4 [15] is used to model the energy deposits produced in the LXe by various backgrounds and the $0 \nu \beta \beta$ signal. The MC simulation propagates the charge deposits through the detector and produces simulated waveforms for each readout channel and event. The MC waveforms are processed using the same analysis framework as the data waveforms. In order to calibrate the detector energy scale and validate the accuracy of the MC simulation, runs are taken using $\gamma$ sources positioned within $10 \mathrm{~cm}$ of the LXe vessel at locations around the cathode plane and at both anode planes. Figure 1 shows the agreement between the MC simulation and data acquired with ${ }^{60} \mathrm{Co},{ }^{226} \mathrm{Ra}$, and ${ }^{228} \mathrm{Th}$ sources.

EXO-200 has previously reported results on a search for $0 \nu \beta \beta[5]$ using $80 \%$ of the data from its first run ("Phase I"), which spans from Sep. 2011 to Feb. 2014. In Feb. 2014, EXO-200 was forced to suspend operations, because of

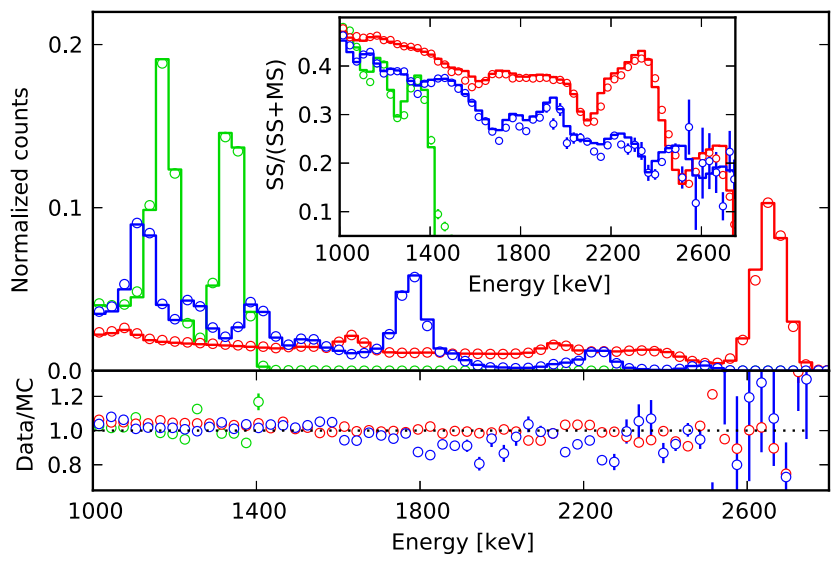

FIG. 1. Comparison between SS events in Phase II data (open markers) and MC simulation (lines) for calibrations using ${ }^{60} \mathrm{Co}$ (green), ${ }^{226} \mathrm{Ra}$ (blue), and ${ }^{228} \mathrm{Th}$ (red) sources positioned near the cathode. The bottom shows the ratio between data and MC. The inset compares the corresponding $\mathrm{SS}$ fraction, $\mathrm{SS} /(\mathrm{SS}+\mathrm{MS})$, for the calibration data and MC. 
accidents at the WIPP facility and recover the Xe from the detector. After access to the experiment was regained in early 2015 , the detector was recommissioned and refilled with LXe in Jan. 2016. Between January and May 2016 the detector was upgraded with new electronics primarily aimed at improving the APD readout noise. In addition, a system was installed to suppress radon in the air gap between the copper cryostat and the lead shield. After installation of this system, direct sampling of the air indicated that the average radon level was reduced by more than a factor of 10 . Fits to the energy and location of backgrounds in physics data also indicated a lower best-fit value for this background component, although more data are required to demonstrate a statistically significant reduction. Finally, the electric field in the drift region of the detector was raised from $380 \mathrm{~V} / \mathrm{cm}$ (cathode voltage, $\left.V_{C}=-8 \mathrm{kV}\right)$ to $567 \mathrm{~V} / \mathrm{cm}\left(V_{C}=-12 \mathrm{kV}\right)$. The datataking run with the upgraded detector began in May 2016 ("Phase II").

The primary goal of the electronics upgrade was to minimize the APD readout noise observed in Phase I. While this noise was accounted for in previous analyses and partially suppressed using a software "denoising" algorithm [16], the hardware upgrade provides substantially improved performance. The effect on the energy resolution is shown in Fig. 2. In Phase I, the SS resolution at the $0 \nu \beta \beta$ decay energy of $Q_{\beta \beta}=2457.83 \pm 0.37 \mathrm{keV}$ [17] after applying the software denoising algorithm is $\sigma / E\left(Q_{\beta \beta}\right)=1.38 \%$, averaged over live time and position. In Phase II, this figure is $1.23 \%$ and its time variation is greatly reduced. These values account for the spatial variation of the resolution, including events taken with the calibration source behind the anodes. Because of the source's proximity to the readout when at the anode, these events present better energy resolution than those in Fig. 2.

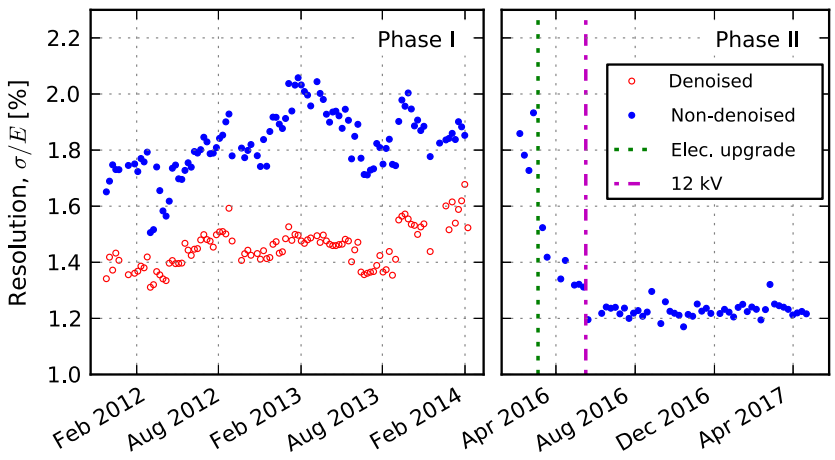

FIG. 2. Measured energy resolution $\sigma / E$ for the $2615 \mathrm{keV}^{208} \mathrm{Tl}$ $\gamma$ line in calibration data taken at the cathode position throughout Phase I and Phase II. The measured resolution before (blue) and after (red) applying the software denoising algorithm in Phase I is shown. The data acquired between restart of operations and the start of Phase II, when $V_{C}$ was raised to $-12 \mathrm{kV}$, were not used in the current analysis.
The selection cuts for this analysis closely follow those used in previous EXO-200 analyses [5]. Both Phase II data and the previously examined Phase I data were "blinded" to remove candidate $0 \nu \beta \beta$ events in the energy region between 2345 and $2570 \mathrm{keV}$. After data quality cuts [8], the total exposure considered here is $596.7 \mathrm{~d}$ and $271.8 \mathrm{~d}$ for Phase I and Phase II, respectively.

Only a fiducial volume (FV) within the detector is considered. The FV selection requires the position of all charge deposits in an event to be reconstructed within a hexagon with apothem of $162 \mathrm{~mm}$ and more than $10 \mathrm{~mm}$ away from the anode and cathode wire planes, as well as from the cylindrical polytetrafluoroethylene (PTFE) reflector inside the field-shaping rings. This FV corresponds to $74.7 \mathrm{~kg}$ of ${ }^{136} \mathrm{Xe}$, i.e., $3.31 \times 10^{26}$ atoms, resulting in a total exposure of $177.6 \mathrm{~kg} \mathrm{yr}$ or $1307 \mathrm{~mol} \mathrm{yr}$. The individual exposure in Phase I and Phase II are $122 \mathrm{~kg} \mathrm{yr}$ and $55.6 \mathrm{~kg}$ yr, respectively, or $898 \mathrm{~mol} \mathrm{yr}$ and $409 \mathrm{~mol} \mathrm{yr}$.

To suppress backgrounds correlated in time, events are required to have only a single reconstructed scintillation signal and to occur $>1 \mathrm{~s}$ from all other reconstructed events. The corresponding $0 \nu \beta \beta$ signal reconstruction efficiency is found to be consistent between phases within errors, $82.4 \pm 3.0 \%(80.8 \pm 2.9 \%)$ for Phase I (Phase II). The inefficiency is dominated by the $1 \mathrm{~s}$ anticoincidence cut and by incomplete reconstruction of $0 \nu \beta \beta$ events with small, separated energy deposits from bremsstrahlung. Its errors are determined from the difference in the observed absolute rate for calibration source data and $\mathrm{MC}$ simulations using the known source activity, and measurements of the individual cut efficiencies for low-background $2 \nu \beta \beta$ events. It includes the estimation of the uncertainty in the FV, 2.8\% in Phase I (2.6\% in Phase II), the dominant term in this error.

This analysis introduces a cut to reduce the rate of background events arising from cosmogenically produced ${ }^{137} \mathrm{Xe}$ [14], which decays via $\beta$ emission with a total energy of $4173 \mathrm{keV}$ [18]. Events in coincidence with the muon veto detector, and depositing energy consistent with the cascade $\gamma$ 's emitted after the neutron capture on ${ }^{136} \mathrm{Xe}$, are used to veto subsequent events in the same TPC half within $19.1 \mathrm{~min}$, corresponding to $5 T_{1 / 2}^{137} \mathrm{Xe}$. This cut was estimated to reduce the number of ${ }^{137} \mathrm{Xe}$ events by $23 \pm 8 \%$, with a loss in exposure of $3.5 \%$ (2.8\%) in Phase I (Phase II). This reduction is consistent with the ${ }^{137} \mathrm{Xe}$ rate entirely attributed to cosmogenic sources [14].

New techniques have been developed to further improve $\gamma$-background rejection among events classified as SS by using the detailed topological information available for each interaction in the TPC. By implementing transverse electron diffusion (coefficient $D_{t}=55 \mathrm{~cm}^{2} / \mathrm{s}$ [11]) and the three-dimensional geometry of the wire planes in the detector model, the number of channels that collect charge signals (denoted as "number of channels") is now accurately simulated. Figure 3(a) shows that SS $\gamma$ backgrounds 

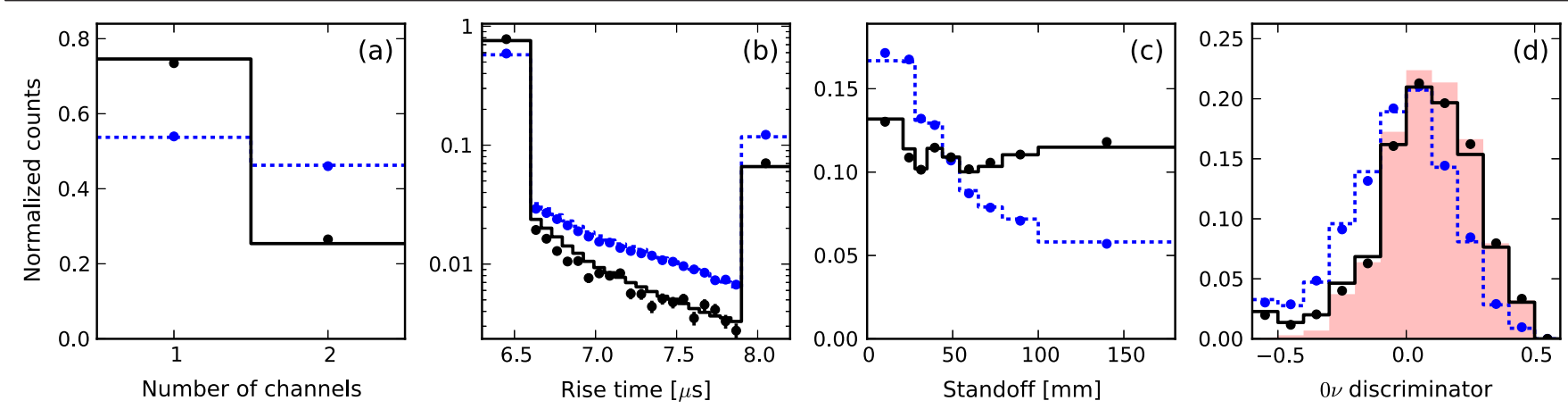

FIG. 3. Comparison between data (dots) and MC simulations (solid and dashed lines) for the three individual variables used in the BDT: (a) number of channels; (b) rise time of the charge pulse; and (c) standoff distance; as well as for the overall discriminator distribution (d). Both source calibration data using the ${ }^{226} \mathrm{Ra}$ source at the cathode (blue dashed) and the background-subtracted $2 \nu \beta \beta$ spectrum from low-background data (black solid) are shown. Only SS events are depicted in the plots. Statistical error bars on the data points are included, but are typically smaller than the marker size. The expected BDT discriminator distribution for a $0 \nu \beta \beta$ signal from MC simulation is indicated by the red filled region. All distributions are normalized by the area, and the edge bins account for overflow.

are more likely to deposit energy on more than one neighboring channel than the $\beta \beta$ signal. In addition, extending this concept to the $z$ direction, the distribution of the rise time of the charge pulse (defined as the time between collection of 5\%-95\% of the total signal) is more likely to extend to large values for $\gamma$ backgrounds relative to $\beta \beta$ events [Fig. 3(b)]. Finally, the "standoff distance," denoting the minimum distance between a cluster and the closest TPC surface, excluding the cathode, is used to constrain backgrounds originating from sources external to the LXe [Fig. 3(c)].

A multivariate discriminator was developed by combining these topological variables in a boosted decision tree (BDT) using the TMVA software package [19]. The separation between SS $0 \nu \beta \beta$ and the most prominent $\gamma$ backgrounds $\left({ }^{238} \mathrm{U},{ }^{232} \mathrm{Th}\right.$, and $\left.{ }^{60} \mathrm{Co}\right)$ was maximized using a subset of the MC simulation. Its performance was then tested on a statistically independent MC data set. Agreement between data and MC simulation for calibration sources for both the BDT and its constituent variables was used to validate its performance for the main backgrounds with high statistics, while the corresponding distributions for signal-like events were investigated using a pure sample of $2 \nu \beta \beta$ SS events with energy near the $Q_{\beta \beta}$. The ranked importance of the individual discriminator variables - defined as the weighted fraction of decision tree cuts for which each variable was used - was found to be $42 \%, 39 \%$, and $19 \%$ for the rise time, standoff distance, and number of channels, respectively.

Figure 3 shows a comparison between the simulated and observed data distributions for calibration sources, and for the measured background-subtracted $2 \nu \beta \beta$ distribution. Overall, the data and MC distributions for the input variables and the overall discriminator agree to better than $10 \%$ at every bin. The detailed binning and range used for each variable was optimized to minimize systematic errors arising from imperfections in the MC simulation, while maintaining as much discriminating power as possible.
As described below, the systematic errors resulting from the differences between the data and MC distributions are evaluated using toy MC studies. These residual differences contribute a subdominant uncertainty to the backgrounds and signal efficiency.

To search for a $0 \nu \beta \beta$ signal, the Phase I and Phase II data are separately fit to models using a binned maximumlikelihood (ML) fit. These models consist of the $0 \nu \beta \beta$ signal and backgrounds originating from the detector and surrounding materials. The background model closely follows that used in previous EXO-200 analyses [5]. The shape of the spectrum for each of the fit observables is determined from the MC simulation for each background and signal component. The energy spectra for the SS and MS data are fit simultaneously, and unlike the previous analysis of Phase I data [5], the BDT variable (including the standoff variable) is added as a fit dimension for the SS data. Toy studies indicated that the addition of the BDT or standoff to the MS fit did not enhance sensitivity for this search. Systematic errors are included in the ML fit as nuisance parameters, constrained by normal distributions. An overall normalization constrained to unity is included to account for the error on the detection efficiency.

The balance between SS and MS events, parametrized by the "SS fraction," is allowed to vary around the expected value from MC simulations for each component within a systematic error. This error was determined by comparing the SS fraction for source calibration data and $\mathrm{MC}$ simulations, as shown in the inset to Fig. 1. Averaging over all calibration positions acquired throughout Phase I (Phase II) gives a relative error on the SS fraction of 5.0\% (8.8\%). An 85\% correlation between the SS fractions of the $\gamma$-like components is included in the constraint, justified by similar levels observed in calibration source data.

Since the ML fit relies on accurately modeling the shapes of the various background components, the impact of shape differences between data and MC simulations was 
TABLE I. Systematic errors on the determination of the number of events near $Q_{\beta \beta}$.

\begin{tabular}{lcc}
\hline \hline Source & Phase I & Phase II \\
\hline Signal detection efficiency & $3.0 \%$ & $2.9 \%$ \\
\hline Background errors & & \\
$\quad$ Spectral shape agreement & $2.1 \%$ & $1.7 \%$ \\
$\quad$ Background model & $5.6 \%$ & $5.9 \%$ \\
$\quad$ Energy scale and resolution & $1.5 \%$ & $1.2 \%$ \\
Total & $6.2 \%$ & $6.2 \%$ \\
\hline \hline
\end{tabular}

investigated for each fit observable (see Figs. 1 and 3). In these studies, the shapes of the $\gamma$-originated background components are corrected by using the residual differences between calibration source data and simulation, while the shapes of the SS $\beta$ components are corrected by using the residual differences of the measured background-subtracted $2 \nu \beta \beta$ spectrum. A large number of simulated data sets were drawn from the best-fit background model using the corrected probability density functions, and were fit with the original simulated shapes. The resulting bias between the fitted and true value of backgrounds near $Q_{\beta \beta}$ is included as an additional systematic error on the normalization of the background components. Toy studies indicate that these shape errors are $2.1 \%$ (1.7\%) for Phase I (Phase II). The contribution to this error caused by spatial and temporal energy resolution variations that are not fully accounted for by the $\mathrm{MC}$ simulation was determined to be $1.5 \%(1.2 \%)$ in Phase I (Phase II).

$\mathrm{U}$, Th, and Co background components simulated at locations different from the default ones were individually inserted into the fit, and the resulting variation in the number of expected events near $Q_{\beta \beta}$ was determined. These studies estimate the error due to uncertainty in the location of the background model components to be $5.6 \%$ (5.9\%) in Phase I (Phase II). All sources of systematic uncertainty on the background model near $Q_{\beta \beta}$ are treated as uncorrelated and result in a total error of $6.2 \%$ for both Phase I and Phase II, as summarized in Table I.

Two final constraints on the measured radon concentration in the LXe and relative rate of cosmogenically produced backgrounds were included in the fit, but verified to be unchanged from previous analyses [5] for both Phase I and Phase II.

The analysis further accounts for a possible difference in the reconstructed energy for $\beta$-like events $E_{\beta}$, relative to the energy scale determined from the $\gamma$ calibration sources $E_{\gamma}$. This difference is expressed through a multiplicative constant $B$ that scales the energy for all $\beta$-like components, $E_{\beta}=B E_{\gamma}$, which is allowed to float freely in the fit. $B$ is highly constrained by the $2 \nu \beta \beta$ spectrum, and consistent with unity to the subpercent level.

After "unblinding" the combined data set, no statistically significant evidence for $0 \nu \beta \beta$ was observed. A lower limit on the half-life of $T_{1 / 2}>1.8 \times 10^{25} \mathrm{yr}$ at the
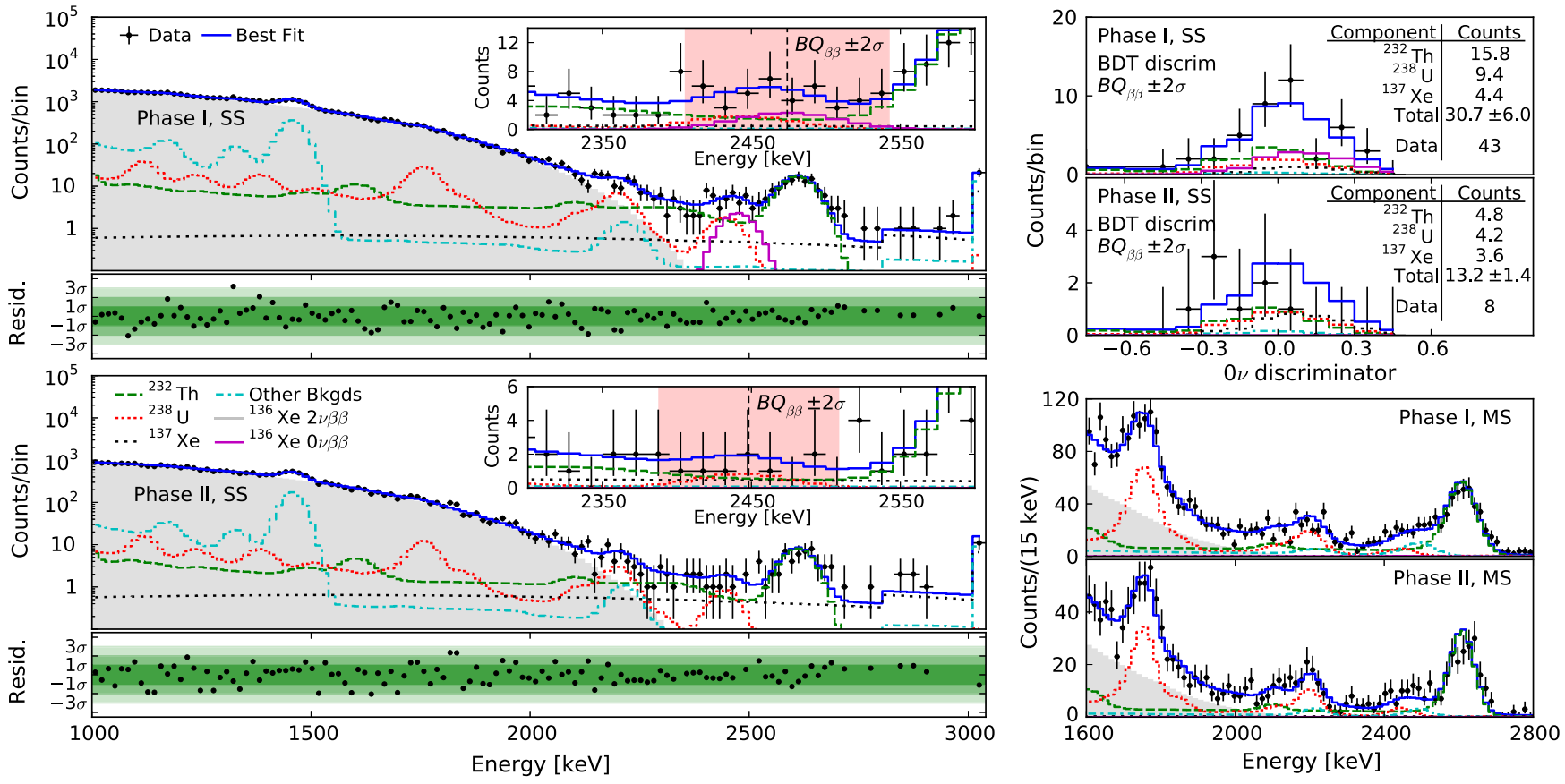

FIG. 4. Best fit to the low-background data SS energy spectrum for Phase I (top left) and Phase II (bottom left). The energy bins are 15 and $30 \mathrm{keV}$ below and above $2800 \mathrm{keV}$, respectively. The inset shows a zoomed-in view around the best-fit value for $B Q_{\beta \beta}$. Top right: Projection of events within $B Q_{\beta \beta} \pm 2 \sigma$ on the BDT fit dimension. Bottom right: MS energy spectra above the ${ }^{40} \mathrm{~K} \gamma$ line. 
90\% confidence level (CL) was derived from the ML fits after profiling over nuisance parameters. The data from each phase are fit separately and the profiles added together considering the difference in live time and signal detection efficiency. No correlation was considered between these two profiles. This conservative assumption was estimated to negligibly change the expected sensitivity. The profilelikelihood distribution was determined from toy $\mathrm{MC}$ simulations, following the same procedure to combine phases, and found to be in good agreement with Wilks's theorem [20,21]. Under the assumption that neutrinos are Majorana particles, this corresponds to an upper limit on the Majorana neutrino mass, $\left\langle m_{\beta \beta}\right\rangle<(147-398) \mathrm{meV}$ [2], using the nuclear matrix elements of [22-26] and phase space factor from Ref. [27]. The best-fit value for the $0 \nu \beta \beta$ component is consistent with the null hypothesis at $1.5 \sigma$, corresponding to a $p$ value of 0.12 .

The results of the ML fits are presented in Fig. 4. The measured $2 \nu \beta \beta$ rates were found to be consistent with Ref. [8]. The best-fit contributions from the primary background components within $B Q_{\beta \beta} \pm 2 \sigma$ are summarized in the inset table in Fig 4 (top right). The best-fit total event rate is $(1.5 \pm 0.3) \times 10^{-3} \mathrm{~kg}^{-1} \mathrm{yr}^{-1} \mathrm{keV}^{-1}$ $\left[(1.6 \pm 0.2) \times 10^{-3} \mathrm{~kg}^{-1} \mathrm{yr}^{-1} \mathrm{keV}^{-1}\right]$ when normalized to the full mass including all Xe isotopes for Phase I.

The median 90\% CL sensitivity was estimated from toy MC studies to be $3.7 \times 10^{25} \mathrm{yr}$. This represents a factor of 2 improvement over the previous EXO-200 search [5]. In comparison to fits using the energy spectra and SS and MS classification alone, or with the addition of only the standoff distance, the use of the BDT discriminator provides a $\sim 15 \%$ increase in sensitivity.

The individual Phase I and Phase II data set lower limits of $1.0 \times 10^{25} \mathrm{yr}$ and $4.4 \times 10^{25} \mathrm{yr}$ at the $90 \% \mathrm{CL}$, respectively, with corresponding median sensitivity of $2.9 \times 10^{25} \mathrm{yr}$ and $1.7 \times 10^{25} \mathrm{yr}$. Because of the detector upgrades and improved topological discrimination described here, the Phase II sensitivity from this analysis is already comparable to that of the previous EXO-200 $0 \nu \beta \beta$ search [5] with an exposure that is half the size. The combined analysis of the Phase I and Phase II data provides one of the most sensitive searches for $0 \nu \beta \beta$ for any isotope [6,7] to date. Further operation of the upgraded detector is expected to continue improving sensitivity to $0 \nu \beta \beta$, and holds promise for $\mathrm{nEXO}$ [28], a tonne-scale LXe TPC being designed to reach half-life sensitivity of $\sim 10^{28} \mathrm{yr}$.

EXO-200 is supported by DOE and NSF in the U.S., NSERC in Canada, SNF in Switzerland, IBS in Korea, RFBR in Russia, DFG in Germany, and CAS and ISTCP in China. EXO-200 data analysis and simulation uses resources of the National Energy Research Scientific Computing Center (NERSC). We gratefully acknowledge the KARMEN Collaboration for supplying the cosmic-ray veto detectors, and the WIPP for their hospitality.
* Corresponding author.

licciard@triumf.ca

†Permanent address: King Abdulaziz City for Science and Technology, Riyadh, Saudi Arabia.

${ }^{\ddagger}$ Present address: P.N. Lebedev Physical Institute of the Russian Academy of Sciences, Moscow, Russia.

${ }^{\S}$ Present address: University of Idaho, Moscow, Idaho, USA. "Present address: Argonne National Laboratory, Argonne, Illinois, USA.

"Present address: SLAC National Accelerator Laboratory, Menlo Park, California, USA.

** Present address: Pacific Northwest National Laboratory, Richland, Washington, USA.

[1] W. H. Furry, Phys. Rev. 56, 1184 (1939).

[2] S. Dell'Oro, S. Marcocci, M. Viel, and F. Vissani, Adv. High Energy Phys. (2016) 2162659.

[3] C. Patrignani et al. (Particle Data Group), Chin. Phys. C 40, 100001 (2016).

[4] J. Engel and J. Menéndez, Rep. Prog. Phys. 80, 046301 (2017).

[5] J. B. Albert et al. (EXO-200 Collaboration), Nature (London) 510, 229 (2014).

[6] A. Gando et al. (KamLAND-Zen Collaboration), Phys. Rev. Lett. 117, 082503 (2016); 117, 109903(E) (2016).

[7] M. Agostini et al. (GERDA Collaboration), Nature (London) 544, 47 (2017).

[8] J. B. Albert et al. (EXO-200 Collaboration), Phys. Rev. C 89, 015502 (2014).

[9] M. Auger et al. (EXO-200 Collaboration), J. Instrum. 7, P05010 (2012).

[10] R. Neilson et al. (EXO-200 Collaboration), Nucl. Instrum. Methods Phys. Res., Sect. A 608, 68 (2009).

[11] J. B. Albert et al. (EXO-200 Collaboration), Phys. Rev. C 95, 025502 (2017).

[12] E. Conti et al., Phys. Rev. B 68, 054201 (2003).

[13] 3M HFE-7000, http://www.3m.com.

[14] J. B. Albert et al. (EXO-200 Collaboration), J. Cosmol. Astropart. Phys. 04 (2016) 029.

[15] J. Allison et al., IEEE Trans. Nucl. Sci. 53, 270 (2006).

[16] C. G. Davis et al. (EXO-200 Collaboration), J. Instrum. 11, P07015 (2016).

[17] M. Redshaw, E. Wingfield, J. McDaniel, and E. G. Myers, Phys. Rev. Lett. 98, 053003 (2007).

[18] E. Browne and J. Tuli, Nucl. Data Sheets 108, 2173 (2007).

[19] A. Hoecker et al., Proc. Sci., ACAT2007 (2007) 040.

[20] S. S. Wilks, Ann. Math. Stat. 9, 60 (1938).

[21] G. Cowan, Statistical Data Analysis (Clarendon Press, Oxford, 1998).

[22] J. Barea, J. Kotila, and F. Iachello, Phys. Rev. C 91, 034304 (2015).

[23] T. R. Rodriguez and G. Martínez-Pinedo, Phys. Rev. Lett. 105, 252503 (2010).

[24] J. Engel, F. Šimkovic, and P. Vogel, Phys. Rev. C 89, 064308 (2014).

[25] J. Menéndez, A. Poves, E. Caurier, and F. Nowacki, Nucl. Phys. A818, 139 (2009).

[26] M. T. Mustonen and J. Engel, Phys. Rev. C 87, 064302 (2013).

[27] J. Kotila and F. Iachello, Phys. Rev. C 85, 034316 (2012).

[28] J. B. Albert et al. (nEXO Collaboration), arXiv:1710.05075. 